Merkel cell carcinoma (MCC) is a rare but very aggressive skin cancer that derives from neuroendocrine cells of the skin. Merkel cell carcinoma morbidity has been continuously increasing for the last few years. Increasing reported incidence of MCC is probably connected with increasing occurrence of this kind of malignancy or with development of histological and immunohistochemical methods of sample examinations which have allowed for more precise diagnosis of skin tumor that might have previously not been accurately recognized. Merkel cell carcinoma develops as nodules early recognized as basocellular carcinoma, squamous cell carcinoma, amelanotic melanoma or skin lymphoma. Merkel cell carcinoma can be morphologically similar to skin metastasis as well as mild changes such as lipoma, cysts, fibroma or vessel changes. Accurate diagnosis is very important because it determines successful management and risk of progression of disease. We describe 5 patients with MCC who underwent surgical excision and then, after estimation of stage of disease, complementary treatment. Our observations prove that every tumor with MCC should be cut out with wide margins and regional lymphadenectomy or sentinel node biopsy is compulsory. After cutting out MCC involved-field radiotherapy is necessary and improves prognosis. Presence of metastases in lymphatic nodes is an indication for complementary chemotherapy.

Key words: Merkel cell carcinoma, radiotherapy, malignancy of skin.

\section{Merkel cell carcinoma - description of five cases}

\section{Mariusz Brąclik, Robert Kwiatkowski, Dorota Katarzyna Nenko}

Department of Radiotherapy, Stanislaw Leszczynski Memorial Hospital, Katowice, Poland

\section{Introduction}

Merkel cell carcinoma (MCC) is 50 times less common than malignant skin melanoma. It is a rare but very aggressive skin cancer which derives from neuroendocrine cells of the skin. In the years 1986-2001 MCC morbidity tripled from 0.15 to 0.44 per 100000 people and increased about $8 \%$ per year - SEER research. Frequency of illness has increased during the last years - statistics for the United States in 2007. The incidence of MCC is higher but the development of histological and immunohistochemical diagnosis allows more accurate examination and distinguishing already existing skin tumours which were not correctly identified and diagnosed as MCC in previous years.

Merkel cell carcinoma incidence increases progressively with Caucasian race, older age and having light-complexion skin; however, men more frequently suffer from MCC, especially those who are white and older than 60 [1, 2]. Merkel cell carcinoma occurs frequently in sun-exposed areas of skin, particularly the head, neck and arms. Generally, a red or purple shiny bump is the main reason for consultation in the outpatient clinic. The tumour itself is usually painless, growing gradually but when bigger than $2 \mathrm{~cm}$ causes pain and ulceration $[3,4]$. People with a weak immune system - including those with HIV or those who take immunosuppressive drugs - are more likely to have MCC. A polio virus can also have a connection with MCC [4]. The stage of disease at the time of diagnosis and the age of the patient are the key points for survival. Stage I of the illness is local change without enlargement of the regional lymph nodes. Stage II is when MCC spreads to the lymph node system and stage III is characterized by remote metastasis and qualifies patients for palliative treatment. Merkel cell carcinoma treatment is connected with the stage of disease and consists in cutting out the tumor with a border of normal skin surrounding the nodule. A recommended margin is a range of $3 \mathrm{~cm}$ around the tumor, which is almost impossible taking the head and neck into consideration. Surgical treatment should consist also in sentinel lymph node biopsy and lymph node dissection $[1,5]$. Complementary treatment applies radiotherapy to the tumor bed with margins surrounding normal tissue and if regional lymph nodes are involved, also those in the lymphatic system. Patients with metastases to lymph nodes additionally receive chemotherapy based on cisplatin $[1,5,6]$. Combination of complete tumor resection and involved-field radiotherapy decreases the risk of local relapse and increases the period free from disease. Merkel cell carcinoma very often spreads (metastasis) through vessels of the lymphatic system; $30 \%$ of tumours smaller than $2 \mathrm{~cm}$ are connected with regional lymph nodes, which is why sampling or biopsy of the sentinel lymph node during surgery is so important. Presence of micrometastases in lymph nodes or sentinel lymph nodes qualifies them to be cut out and undergo involved-field radiotherapy [7-10].

\section{Description of cases}

\section{Patient 1}

The 70-year-old patient came to the surgery outpatient clinic with a tumor on the skin near to the left knee. During physical examination a pur- 
ple $1 \mathrm{~cm}$ skin tumour was found on the middle part of the left thigh above the knee joint. After preparation of the patient the tumour was removed with the surgery margin. In histological examination there was found: nodule $1.3 \times 1 \mathrm{~cm}$, longitudinal margin I-7 mm, longitudinal margin $11-4 \mathrm{~mm}$, and a deep, very narrow margin. The whole nodule was cut out.

Because of the narrow deep margin and lack of estimation about the sentinel lymph node the patient was asked to see the surgeon again to undergo a radicalization procedure - cutting out the tumour bed, and removal of regional lymph nodes.

The postoperative bed with margin of surrounding normal tissue was removed with left inguinal lymphadenectomy. In the obtained material no cancer was found or micrometastases in inguinal lymph nodes. The decision about radiation therapy was made because of the characteristics of MCC as a bad prognosis cancer, with high risk of local relapse and distant metastasis through the lymphatic system. The plan was to deliver the dose of 60 Gy on the postoperative bed in conformal radiotherapy and 50 Gy on margins of $5 \mathrm{~cm}$ normal tissues during 6 weeks of treatment. No complications were found during radiotherapy. After one month following treatment we observed a smooth brown scar, dry skin and a little swelling of the left leg. The left inguen was without pathological resistance. The patient said that 2 weeks earlier the radiation-induced reaction had become stronger together with gall and damp desquamate. Antibiotic ointment and moisturization of the skin cured the situation. The patient was placed under the supervision of an oncologist, performing physical and imaging examinations every 3 months. One year after the procedure screening did not reveal any metastases or local recurrences. The recommended screening examination, which included physical examination, $X$-rays of chest, ultrasonography of abdomen and regional lymph node and laboratory tests, was performed every 6 months.

\section{Patient 2}

After left buttock tumour removal a 55-year-old female patient was referred to oncology with histological diagnosis: MCC; CK (+) chromogranin (+), HMB45 (-), LCA (-). The presence of cancer in the cut margin was found. The woman was suggested to have the tumour bed cut out with safe margins. After the procedure histological diagnosis was: all margins free from cancer. Close to the scar a small ( $2 \mathrm{~mm})$ focus of malignant tumour was visible. $X$-ray of the chest and ultrasonography of the abdominal cavity were performed and no distant metastasis or enlargement of lymph nodes was found. Diffuse disease was excluded and the patient was qualified for complementary involved-field radiotherapy. Conformal treatment used irradiation of the postoperative scar with a $5 \mathrm{~cm}$ margin to the dose of $66 \mathrm{~Gy}$. It took 6 weeks administering 2 Gy every day without Saturdays and Sundays. Complications were not observed. At the end of radiotherapy we observed acute side-effects after radiation on the skin as reddening and swelling. These side-effects disappeared in 4 weeks of symptomatic cure. Screening of chest and stomach, and ultrasonography of abdominal cavity did not reveal any metastasis. No signs of local recurrences were found in the postoperative scar.

\section{Patient 3}

A 76-year-old female patient was admitted to the oncological outpatient clinic after $1.5 \times 1 \mathrm{~cm}$ tumour removal from the gluteal region. Histological examination results: MCC, CK (+), chromogranin (+), S100 (-). There was no cancer found in any surgical margins. Screening of the chest and abdominal cavity was performed and no signs of cancer dissemination were found. She was qualified for complementary radiotherapy of the postoperative scar and tumour bed with $5 \mathrm{~cm}$ margins. The dose of $66 \mathrm{~Gy}$ in 33 fractions was scheduled in 6 weeks. During treatment no acute side-effects of radiation were observed. Two weeks after radiotherapy reddening and swelling of skin occurred but disappeared after local cure. Three months later, screening of the chest and abdominal cavity was performed but no metastases were found. Physical examination of the postoperative scar did not show any recurrences. The patient is still under the supervision of an oncologist.

\section{Patient 4}

A 59-year-old male patient was referred to oncology with MCC found in a tumour removed from the left lumbar side. The histological results: MCC CK (+), chromogranin (+), synaptophysin (+), TTF-1 (-), LCA (-), Ki 67 (+) 50\%. Malignant changes were present in all limits of the cut. The patient qualified for chemotherapy and involved-field radiotherapy on the scar with margins. Six PE series were given and then there was planned $60 \mathrm{~Gy}$ in 30 fractions during 6 weeks on the scar with safety margins. During and after radiotherapy no complications were observed. Reddening occurred as an acute side-effect of radiation. The patient was under permanent oncologist control. During the second check-up - 6 months after radiotherapy - physical examination revealed enlargement of inguinal lymph nodes on the left side and ultrasonography showed enlargement of iliac lymph nodes also on the left side. Biopsy of those lymph nodes revealed metastasis with cells CK (+) chromogranin (+). It was recognized as progression of the malignant disease. Palliative radiotherapy on the small pelvis was done with a dose of $20 \mathrm{~Gy}$ in 5 fractions and 6 series of chemotherapy DDP + VP were applied. The next check-up revealed expansion of right inguinal lymph nodes and histological sampling confirmed the spread of MCC. The patient was qualified for palliative radiotherapy of right inguinal lymph nodes. Computed tomography scan of the chest and abdominal cavity was performed with the result of no metastasis and expansion of lymph nodes. However, magnetic resonance examination showed metastasis in the pelvis minor lymph. Finally, PETCT examination excluded all metastases except those mentioned above.

\section{Patient 5}

A 64-year-old male patient was referred to the oncological outpatient clinic after partial removal of an ulcerate tumour on the left side of the forehead. Histological results: MCC, Excisio partialis, CK (+), synaptophysin (+), chromogranin $( \pm)$ EMA (-). Screening did not confirm any metastasis. Because of the non-radical nature of the operation, the patient 
was qualified for palliative radiotherapy with 20 Gy in 5 fractions. The check-up revealed regression of ulceration and healing of the tissues around the ulceration. It was decided to apply the next 20 Gy dose in 5 fractions. In another 3 months CT scanning was performed and revealed broad, irregular tissues around the forehead-temple suggesting local relapse of cancer.

\section{Summary}

Merkel cell carcinoma is a very rare skin cancer, but we can observe steady growth of incidence, even $8 \%$ every year. Merkel cell carcinoma clinically is a small tumour which frequently is overlooked and diagnosed at a later stage. Because of unusual appearance it is often excised in surgery or dermatology without margins and an appropriate diagnostic procedure of regional lymph. Negligence of treatment and too long tumour observation lead to development of cancer and make radical treatment impossible.

The main treatment of MCC is surgical operation with cutting of changes with widely negative margins. Removal of the changes is so important because of cancer spreading through skin lymph vessels. The tendency of MCC for metastasis through the lymphatic system inclines the surgeon to localize the nearest group of lymph nodes and possibly to remove the sentinel lymph node or perform regional lymphadenectomy. Histological examination is always a must after removal of skin tumour. Merkel cell carcinoma does not have a special appearance so microscope and immunohistochemical examinations confirm the diagnosis. Affirmation of MCC leads to cutting the tumour site together with safe margins $[8,9]$. In our own observations we found out that skin malignant tumour is often resected without margins.

Patients who underwent procedures of cutting out the tumour site with margins were free from metastasis in lymph nodes and recurrences in the postoperative scar. In 2 patients in whom no re-operation was performed metastases to lymph nodes were observed despite complementary treatment of radio- and chemotherapy being applied. One of our patients who underwent partial removal of a tumour around the forehead was treated with palliative radiotherapy but to the rest radiotherapy was applied as a complementary therapy with a dose of 60-66 Gy in 7 weeks. In one patient, a second operation of removal of the tumour site with margins was not carried out because non-radical first operative procedure cancer progression and metastasis to lymph nodes appeared. Merkel cell carcinoma can be wrongly diagnosed as basal cell carcinoma, squamous cell carcinoma, amelanotic melanoma or skin lymphoma. Merkel cell carcinoma can be morphologically similar to skin metastasis as well as mild changes of lipoma, cysts, fibroma or vessel changes [1]. Merkel cell carcinoma prognosis depends on progression of disease so it is important to diagnose it fast and provide appropriate treatment even faster. In spite of our observations concerning just a few patients, we are convinced of the necessity of a second procedure of cutting the tumour bed with wider margins. Involved-field radiotherapy is the next very important element of our observations. After partial removal of a lesion (ulcerating tumour around the forehead) palliative radiotherapy as a complementary therapy was used. Treat- ment caused decrease of ulceration and partial healing of the lesion. Because the procedure was non-radical there was local progression and development of tumour but even small progress and effect supports the radiotherapy treatment.

Each tumour removal should be followed by histological examination and any suspicion of MCC requires immunohistochemical examinations which result in fewer diagnostic mistakes. Confirmation of MCC should put into consideration the next procedure of removal of the postoperative bed and safe margins. Complementary treatment performs a serious role and its applications depend on the stage of the disease. Screening of the chest and abdominal cavity play an important role in exclusion of distant metastasis. Any case of MCC suspicion should be followed by sentinel lymph node biopsy and regional lymphadenectomy. After elimination of distant metastasis and involved lymph nodes postoperative radiation therapy should be applied.

The authors declare no conflict of interest.

\section{References}

1. Calder KB, Smoller BR. New insights into Merkel cell carcinoma. Adv Anat Pathol 2010; 17: 155-61.

2. Clark JR, Veness MJ, Gilbert R, O'Brien CJ, Gullane PJ. Merkel cell carcinoma of the head and neck: is adjuvant radiotherapy necessary? Head Neck 2007; 29: 249-57.

3. Kaae J, Hansen AV, Biggar RJ, Boyd HA, Moore PS, Wohlfahrt J, Melbye M. Merkel cell carcinoma: incidence, mortality and risk of other cancers. J Natl Cancer Inst 2010; 102: 793-801.

4. Zampetti A, Feliciani C, Massi G, Tulli A. Updated review of the pathogenesis and management of Merkel cell carcinoma. J Cutan Med Surg 2010; 14: 51-61.

5. Rosado P, Junquera L, Vivanco B, García-Consuegra L, Gallego L Merkel cell carcinoma: our experience in this rare pathology. Med Oral Patol Oral Cir Bucal 2011; 16: 736-9.

6. Ziółkowska E, Pietrusińska E, Biedka M, Weiss-Rostkowska W, Makarewicz R. Rak z komórek Merkla - neuroendokrynny rak skóry, postępowanie. Onkol Prakt Klin 2008; 4: 141-4.

7. Fang LC, Lemos B, Douglas J, lyer J, Nghiem P. Radiation monotherapy as regional treatment for lymph node-positive Merkel cell carcinoma. Cancer 2010; 116: 1783-90.

8. Gonzalez RJ, Padhya TA, Cherpelis BS, Prince MD, Aya-Ay ML, Sondak VK, Cruse CW, Zager JS. The surgical management of primary and metastatic Merkel cell carcinoma. Curr Probl Cancer 2010; 34: 77-96.

9. Stokes JB, Graw KS, Dengel LT, Swenson BR, Bauer TW, Slingluff CL $J \mathrm{r}$, Ledesma EJ. Patients with Merkel cell carcinoma tumors $\leq 1.0 \mathrm{~cm}$ in diameter are unlikely to harbor regional lymph node metastasis. J Clin Oncol 2009; 27: 3772-7.

10. Rao NG. Review of the role of radiation therapy in the management of Merkel cell carcinoma. Curr Probl Cancer 2010; 34: 108-17.

\section{Address for correspondence}

Mariusz Brąclik MD, PhD

Department of Radiotherapy

Stanislaw Leszczynski Memorial Hospital

Raciborska 27

40-074 Katowice, Poland

e-mail: braclik@o2.pl

Submitted: $\quad 27.12 .2011$

Accepted: $\quad$ 18.04.2012 